\title{
Influence of grid distribution on CFD model of compressible flow inside the primary nozzle and mixing chamber used in refrigeration application
}

\author{
Natthawut Ruangtrakoon ${ }^{1, *}$, and Eakarach Bumrungthaichaichan ${ }^{2}$ \\ ${ }^{1}$ Department of Mechanical Engineering, Faculty of Engineering, King Mongkut's Institute of Technology Ladkrabang, Bangkok, \\ Thailand \\ ${ }^{2}$ Department of Chemical Engineering, Faculty of Engineering, King Mongkut's Institute of Technology Ladkrabang, Bangkok, \\ Thailand
}

\begin{abstract}
In this study, the influence of grid distribution on CFD model of the primary nozzle and mixing chamber used in refrigeration application was primarily investigated. The only one geometry of primary nozzle and mixing chamber was modeled. The two different grid distributions, fine near-wall grid and regular grid with the identical total grid number, were simulated to investigate the flow phenomena inside the considered system. The appropriate boundary conditions and numerical methods were carefully employed. The simulated entrainment ratios obtained by two different grid arrangements were validated by comparing with the reliable experimental data. The results revealed that the Mach number distributions of these models were different. Further, the outlet total pressure predicted by fine near-wall grid was about $1.3 \%$ higher than that obtained by regular grid.
\end{abstract}

\section{Introduction}

A steam jet refrigeration system can convert waste heat (low-grade thermal energy), that is rejected from many industrial processes, to useful refrigeration. So, the electrical energy used in air-conditioning system can be reduced. Another advantageous point is that water, the most environmental friendly substance, can be used as the refrigerant in the system.

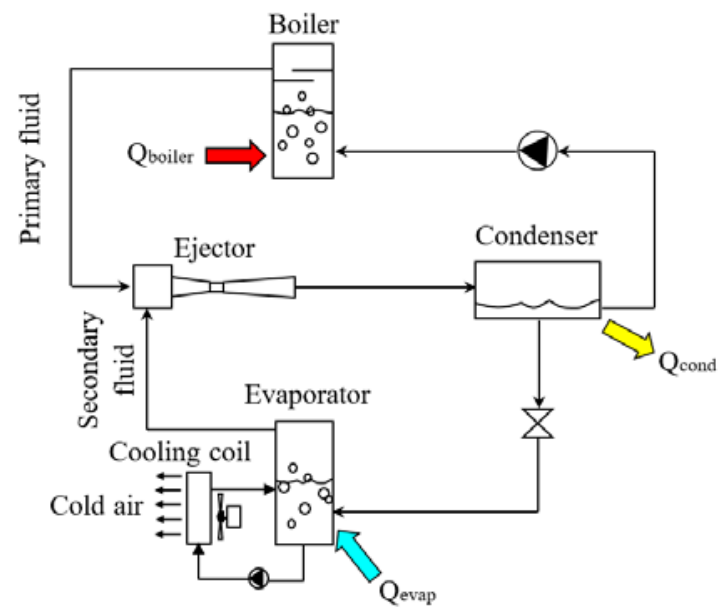

Fig. 1. Schematic view of a steam jet refrigeration cycle

Fig. 1 presents a schematic view of a steam ejector refrigeration system. The stream ejector, which is used as the thermal compressor for steam jet refrigeration system, provides four main parts, including primary nozzle, mixing chamber, throat, and subsonic diffuser, as shown in Fig. 2.

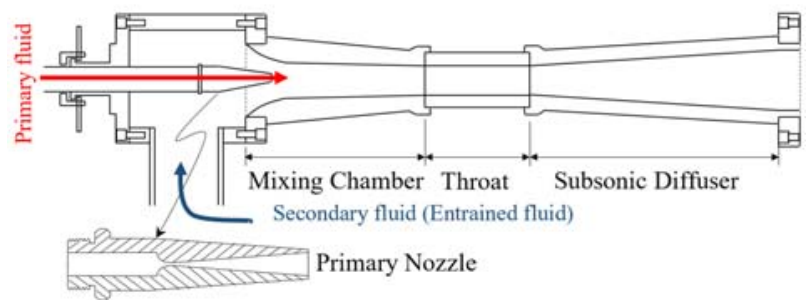

Fig. 2. Schematic of steam ejector

The performance of steam jet refrigeration system can be defined in term of the coefficient of performance (COP) for steam jet refrigeration as depicted in Eq. (1).

$$
\mathrm{COP}=R_{m} \cdot \frac{h_{g-\text { evap }}-h_{f-\text { con }}}{h_{g-\text { boiler }}-h_{f-\text { con }}}
$$

where $R_{m}$ is the entrainment ratio of the steam ejector, which can be defined as a ratio of mass flow rate of primary fluid to mass flow rate of secondary fluid and $h_{g}$ and $h_{f}$ respectively are enthalpy of vapor and liquid.

Since, the enthalpy change at the boiler is not much different from the enthalpy change at the evaporator. Then, it can be assumed that: 


$$
\mathrm{COP} \approx R_{m}
$$

So, the performance of jet refrigeration system depends almost on the performance of steam ejector. There are two parameters used to define the steam ejector performance, which are entrainment ratio as mentioned earlier and critical discharge pressure. In term of critical discharge pressure, it can be used to define the highest possible pressure of mixed fluid which the steam ejector can discharge to the condenser.

Over the past decades, many researchers have been attempted to improve the performance of steam jet refrigeration system by considering the experimental investigations [1,2]. Moreover, the computational fluid dynamics (CFD) is rapidly developed. It can be used to explain the mixing phenomenon inside the ejector. The predicted results of CFD were found to agree well with experimental values [3-5]. That is, CFD technique can be used to accurately predict the performance of the steam jet refrigeration system.

In order to improve the CFD results, the effect of grid distribution on CFD compressible flow model was studied. The two principle components of steam ejector (primary nozzle and mixing chamber) were primarily selected as the computational domain. The two different grid distributions were constructed and the simulated entrainment ratios obtained by these models were validated by comparing with the reliable experimental data. The boundary conditions as well as the numerical models were carefully employed. From this preliminary CFD study, the understand of the influence of grid distribution on flow behavior inside primary nozzle and mixing chamber, which directly affects on the performance of steam ejector jet refrigeration system, was obtained.

\section{Description of CFD modelling}

\subsection{Computational domain}

The dimensions of primary nozzle and mixing chamber used in the calculation domain as shown in Fig. 3 were similar to the steam ejector of Ruangtrakoon et al. [3], which based on Engineering Sciences Data Unit (ESDU) design. The diameters of primary nozzle throat $(d)$ and exit $(D)$ are $1.7 \mathrm{~mm}$ and $7.6 \mathrm{~mm}$, respectively. The divergent angle of primary nozzle is $10^{\circ}$. This allows the nozzle to produce the Mach number of 4 at the primary nozzle exit plane.

\subsection{Grid generation}

The GAMBIT 2.4.6 program was employed to perform the two-dimensional axis-symmetrical solid model and grid generation of the domain. The quadrilateral grids were carefully generated inside the flow domain to achieve the accurate results. The grids inside primary nozzle of these models were different. Whereas, the axial grid sizes of mixing chamber were controlled to be identical. The total number of grids for two different grid distributions were identical $(110,460$ cells). The grid generation of these simulations are shown in Fig. 4.

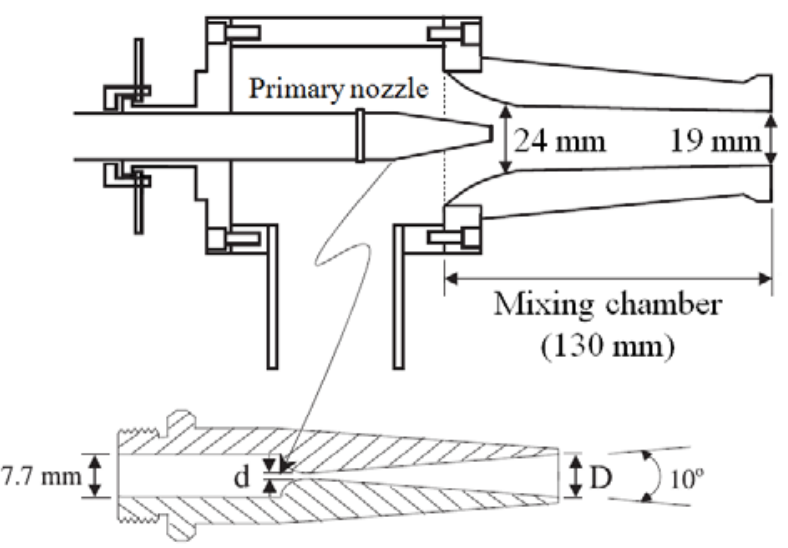

Fig. 3. Schematic of computational domain

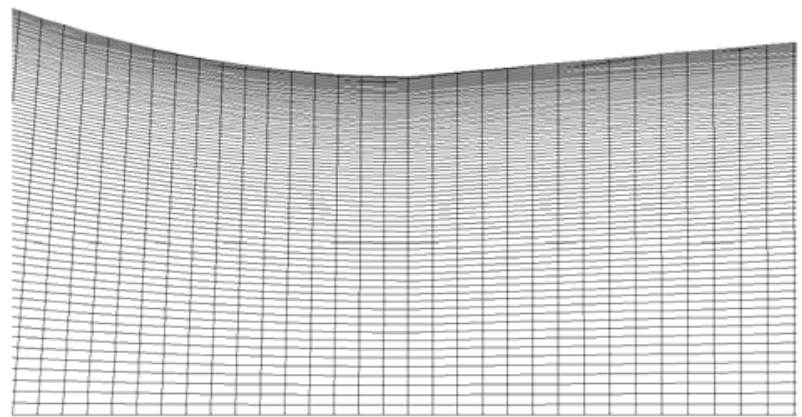

Fine near-wall grid

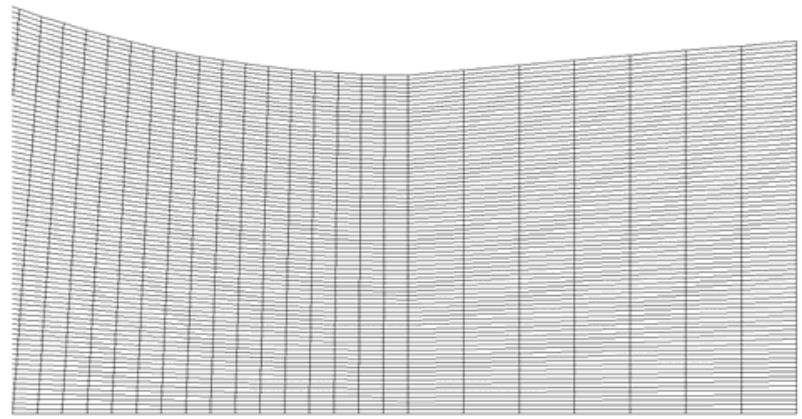

Regular grid

Fig. 4. Grid generation at nozzle throat

\subsection{Governing equations}

For CFD simulations of compressible flows, the steady state flow pattern and turbulence field were obtained by using the Reynolds average equations for conservation of mass and momentum together with the shear stress transport k-omega model (SST-k-omega). The compact form of steady state Reynolds average equation can be written as:

$$
\frac{\partial(\rho \boldsymbol{U} \phi)}{\partial x_{j}}=\frac{\partial}{\partial x_{j}}\left(\Gamma_{\varphi} \frac{\partial \phi}{\partial x_{j}}\right)+S_{\varphi}
$$

where $\boldsymbol{U}$ is mean velocity vector, $\phi$ is a universal dependent variable, $\Gamma_{\varphi}$ is the diffusivity, and $S_{\varphi}$ is the source term. Further, the details of variables for 
continuity equation, momentum equation, and SST-komega are represented in Table 1.

Table 1. Variables for continuity equation, momentum equation, and SST-k-omega [6]

\begin{tabular}{|c|c|c|c|}
\hline Equation & $\phi$ & $\boldsymbol{\Gamma}_{\varphi}$ & $\boldsymbol{S}_{\varphi}$ \\
\hline Continuity & 1 & 0 & 0 \\
\hline Momentum & $U_{i}$ & $\mu$ & $-\frac{\partial P}{\partial x_{i}}+\frac{\partial}{\partial x_{j}}\left(\mu_{t} \frac{\partial U_{i}}{\partial x_{j}}\right)+S_{M, i}$ \\
\hline$k$-transport & $k$ & $\mu+\frac{\mu_{t}}{\sigma_{k}}$ & $G_{k}-Y_{k}+S_{k}$ \\
\hline$\omega$-transport & $\omega$ & $\mu+\frac{\mu_{t}}{\sigma_{\omega}}$ & $G_{\omega}-Y_{\omega}+D_{\omega}+S_{\omega}$ \\
\hline
\end{tabular}

Further, the energy equation was used to achieve the energy distribution inside the system. The steady state energy equation can be written as:

$$
\begin{aligned}
& \frac{\partial(\boldsymbol{U}(\rho E+p))}{\partial x_{j}}=\frac{\partial}{\partial x_{j}}\left(k_{e f f} \frac{\partial T}{\partial x_{j}}-\sum_{j} h_{j} \vec{J}_{j}+\left(\overline{\bar{\tau}}_{e f f} \cdot \boldsymbol{U}\right)\right) \\
& +S_{h}
\end{aligned}
$$

where $E$ is energy, $k_{\text {eff }}$ is effective conductivity, $\vec{J}_{j}$ is the diffusion flux of species $j$, and $S_{h}$ is source term for energy equation.

\subsection{Boundary conditions}

For CFD simulations of compressible flows, FLUENT [7] recommended to specify the zero operating pressure to minimize the errors due to pressure fluctuations. According to Fig. 3, the pressure-inlet and pressureoutlet boundary condition types were adopted at inlet of primary nozzle and mixing chamber and outlet of mixing chamber, respectively. Moreover, water vapor was used as the working fluid and was assumed to be ideal gas.

At primary nozzle inlet, the gauge total pressure and total temperature of primary fluid were $313,201 \mathrm{~Pa}$ and $408 \mathrm{~K}$, respectively. Whereas, for mixing chamber inlet, the gauge total pressure of $873 \mathrm{~Pa}$ and total temperature of $280.5 \mathrm{~K}$ were imposed as the conditions of secondary fluid. At outlet section, the gauge pressure of $611.1 \mathrm{~Pa}$ and total temperature of $300 \mathrm{~K}$ were set. Further, the $1 \%$ turbulence intensity was adopted for all simulations. At the wall, the no-slip boundary condition and adiabatic condition were used.

\subsection{Numerical methods and solution strategy}

In this work, the double precision density-based solver of ANSYS FLUENT program was employed. The implicit formulation with Roe flux-difference splitting scheme was adopted. The spatial discretization scheme for gradient was least squares cell-based. Further, the spatial discretization schemes of flow and turbulence quantities were second order upwind.

Further, in order to obtain the converged solutions, the CFD models were computed until the residuals of all quantities were no longer change.

\section{Results and discussion}

Table 2 represents the model validation between the simulated entrainment ratios and experimental data of Ruangtrakoon et al. [1]. The results showed that the predicted results of these two different grid distributions were in good agreement with experiment. Meaning that, the present CFD models were properly developed.

Table 2. Model validation

\begin{tabular}{|c|c|c|}
\hline \multicolumn{3}{|c|}{ Entrainment ratio $\left(\boldsymbol{R}_{\boldsymbol{m}}\right)$} \\
\hline EXP [1] & Fine near-wall grid & Regular grid \\
\hline 0.358 & 0.364 & 0.362 \\
\hline
\end{tabular}

Fig. 5 shows filled and line contours of Mach number for different grid distributions. According to this figure, the Mach number inside the primary nozzle and mixing chamber obtained by two different grid distributions are slightly different.

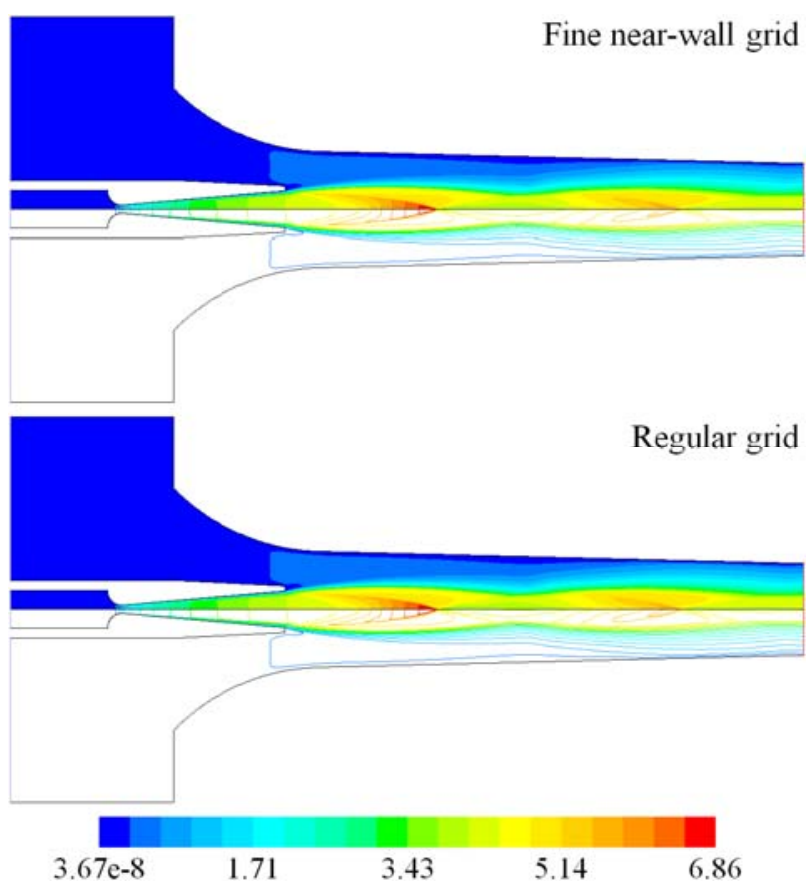

Fig. 5. Filled (top) and line (bottom) contours of Mach number

The difference in Mach number contour of these models is significantly observed at the region near primary nozzle exit as depicted in Fig. 6. From Fig. 6, the appearance of first shock obtained by fine near-wall grid is faster than that predicted by regular grid. This difference may be due to the growth rate of grid at the primary nozzle exit. That is, the growth rate of regular grid was higher than fine near-wall grid, which directly 
degrades the solution accuracy in the region of large gradient [8].

\section{Fine near-wall grid}

Regular grid

Fig. 6. Line contours of Mach number near primary nozzle exit

Further, the difference in flow phenomena inside this considered system can be illustrated by considering the centerline Mach number of two different grid distributions as shown in Fig. 7.
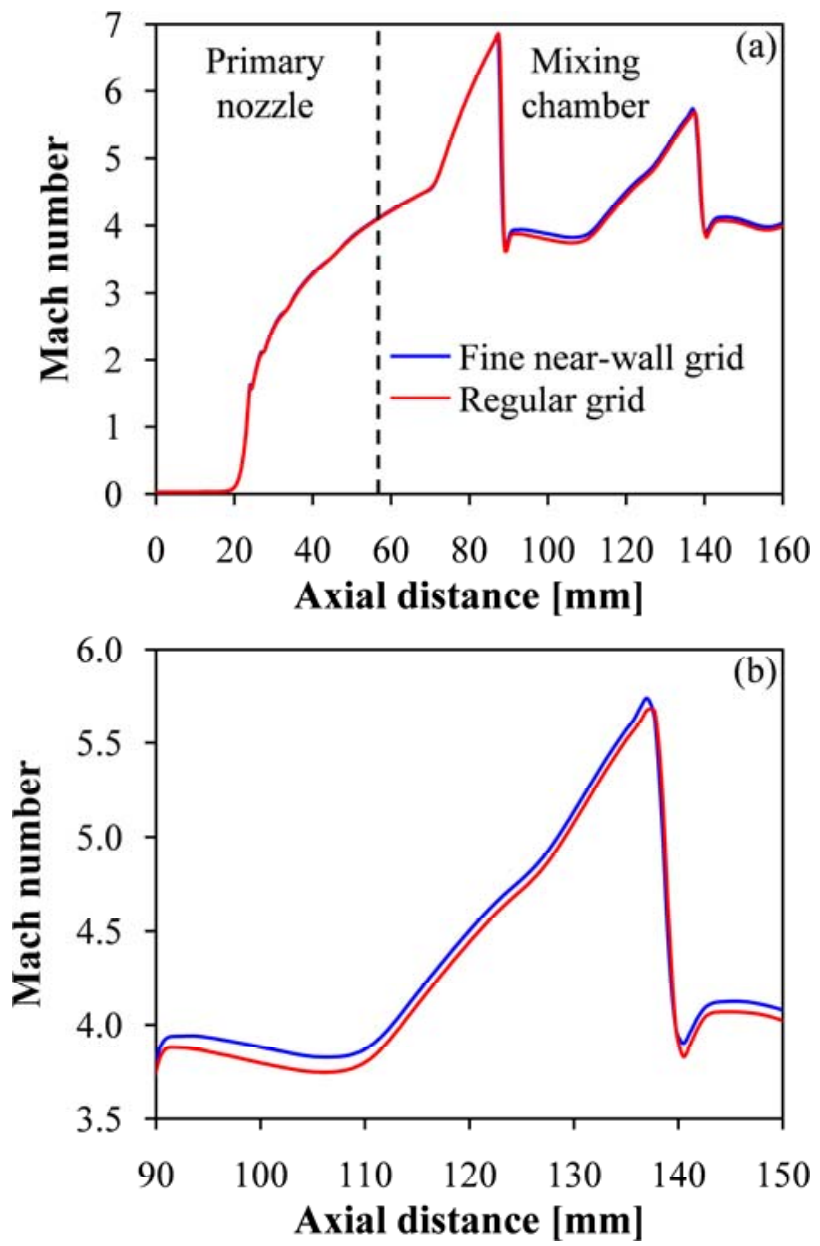

Fig. 7. Mach number profiles: (a) overall (b) enlargement

From Fig. 7, the simulated results show that the centerline Mach number inside the primary nozzle of these two different grids are similar. However, after the primary nozzle exit, the centerline Mach number of fine near-wall grid is higher than that predicted by regular grid, especially for axial distance between $90-160 \mathrm{~mm}$, as shown in Fig. 7(b). Further, the predicted outlet total pressure of fine near-wall grid $(89,640 \mathrm{~Pa})$ is about $1.3 \%$ higher than that predicted by regular grid $(88,507 \mathrm{~Pa})$.

According to these preliminary simulated results, even when the axial grid size in mixing chamber of two different models are identical, the flow patterns inside the mixing chamber are different. Hence, it can be implied that the reason of this difference in flow pattern would be due to the grid distribution inside the primary nozzle. Meaning that, the grids inside the primary nozzle should be carefully generated to achieve the accurate downstream results, such as the location of first shock, Mach number, critical discharge pressure, etc.

\section{Conclusion}

In this work, the effect of grid distribution inside the primary nozzle on downstream phenomena was numerically studied by considering the fine near-wall grid and regular grid. The predicted entrainment ratios of these models were in good agreement with experimental data of Ruangtrakoon et al. [1]. The results revealed that even when the axial grid sizes of mixing chamber for two different models were identical, the Mach number distributions inside these models were different, especially the region near primary nozzle exit, e.g. the appearance of first shock obtained by fine near-wall grid was faster than that predicted by regular grid. The outlet total pressure of these models were also different. So, the grid generation inside primary nozzle should be carefully created. For future work, in order to achieve the comprehensive model for predicting the compressible flow inside the steam ejector, the three-dimensional model of actual configuration should be simulated.

The authors would like to thank College of Advanced Manufacturing Innovation, King Mongkut's Institute of Technology Ladkrabang, Thailand for supporting the ANSYS FLUENT software.

\section{References}

1. N. Ruangtrakoon, S. Aphornratana, T. Sriveerakul, Exp. Therm. Fluid Sci. 35, 676 (2011)

2. F. Li, R. Li, X. Li, Q. Tian, Appl. Therm. Eng. 137, 784 (2018)

3. N. Ruangtrakoon, T. Thongtip, S. Aphornratana, T. Sriveerakul, Int. J. Therm. Sci. 63, 133 (2013)

4. S. Varga, A.C. Oliveira, B. Diaconu, Int. J. Refrig.Rev. Int. Froid 32, 1203 (2009)

5. G. Besagni, F. Inzoli, Appl. Therm. Eng. 117, 122 (2017)

6. ANSYS Inc., ANSYS Fluent Theory Guide: Release 15.0 (ANSYS Inc., USA, 2013)

7. FLUENT Inc., Tutorial 7. Inviscid and Compressible Flow through a Converging-Diverging Nozzle (FLUENT Inc., USA, 2006)

8. S.-E. Kim, T. Barbat, P. Spicka, Meshing and CFD Accuracy (FLUENT Inc., USA, 2005) 